\title{
On the Symmetry and Structure of Cubic Semiconductor Surfaces
}

\author{
S.J. Jenkins* \\ Department of Chemistry, \\ University of Cambridge, Lensfield Road, \\ Cambridge, CB2 1EW, UK \\ (Dated: October 9, 2017)
}

\begin{abstract}
A systematic stereographic approach to the description of surface symmetry and structure, applied previously to face-centred cubic, body-centred cubic and hexagonal close-packed metals, is here extended to the surfaces of diamond-structure and zincblende-structure semiconductors. A variety of symmetry-structure combinations are categorised, and the chiral properties of certain cases emphasised. A general condition for non-polarity in the surfaces of zincblende materials is also noted.
\end{abstract}

* Corresponding Author: sjj24@cam.ac.uk

Keywords: Structure; Symmetry; Stereograms; Diamond; Zincblende; Chirality

\section{INTRODUCTION}

Ten years ago, the present author co-authored a detailed analysis of the symmetry and structure of surfaces belonging to the face-centred cubic, body-centred cubic and hexagonal close-packed metals [1]. The essence of the approach developed in that work may be summarised in three steps: firstly, the presentation, in a stereographic representation, of the improper symmetry elements found at the surface; secondly, the presentation, in a compatible stereographic representation, of certain key structural elements found at the surface; and thirdly, the presentation of a combined stereographic representation of symmetry and structure together. In this way, it was possible not only to identify a variety of surface symmetry and surface structural categories, but also to note which of those categories could apply to a single surface and which are necessarily mutually exclusive. For instance, it was shown for materials taking the $f c c$ structure that only some surfaces featuring low-coordination kink atoms are chiral, whereas all chiral surfaces exhibit kink atoms, and no surfaces with perfectly straight step edges can ever be chiral. Similarly fundamental findings were obtained for the surfaces of materials taking the $b c c$ and hcp structures.

In developing our approach, we were careful to ensure that it would be generally applicable to crystalline materials beyond the three commonly occurring structures considered at the time. Although $f c c, b c c$ and $h c p$ encompass most of the interesting pure metals, one could certainly wish to extend the analysis to include common structures found amongst the semiconductors, such as the diamond structure, the zincblende structure and the wurtzite structure. The first two of these share some features in common with the two cubic structures dealt with in the earlier work, but with additional complexity due to the presence of two atoms within their primitive unit cells - complexity previously encountered only in the hexagonal case. The purpose of the present work is to investigate the surfaces of materials taking these two crystal structures. The wurtzite case is considerably more complex, and consideration of its surfaces will be deferred for the present.

\section{BULK STRUCTURES}

Although the diamond and zincblende bulk crystal structures are very well-known, it will be convenient briefly to summarise the key features prior to our discussion of surface structure and symmetry. Both bulk structures are based upon the $f c c$ Bravais lattice, but unlike the $f c c$ crystal structure they each possess two atoms per primitive unit cell. We shall express the primitive lattice vectors as

$$
\begin{aligned}
& \mathbf{a}_{1}=\frac{1}{2}\left[\begin{array}{lll}
1 & 1 & 0
\end{array}\right] \\
& \mathbf{a}_{2}=\frac{1}{2}\left[\begin{array}{lll}
0 & 1 & 1
\end{array}\right] \\
& \mathbf{a}_{3}=\frac{1}{2}\left[\begin{array}{lll}
1 & 0 & 1
\end{array}\right]
\end{aligned}
$$

and the positions of the atoms relative to each lattice point as

$$
\begin{aligned}
\mathbf{r}_{\alpha} & =\frac{1}{8}\left[\begin{array}{lll}
1 & \overline{1} & \overline{1}
\end{array}\right] \\
\mathbf{r}_{\beta} & =\frac{1}{8}\left[\begin{array}{lll}
1 & 1 & 1
\end{array}\right]
\end{aligned}
$$

where these last are to be interpreted as fractional coordinates in the basis set defined by the primitive lattice vectors. The resulting structure may be represented within either a primitive or a conventional cubic unit cell, as shown in Fig. 1. In the diamond structure, the atoms labelled $\alpha$ and $\beta$ are, of course, identical with one 


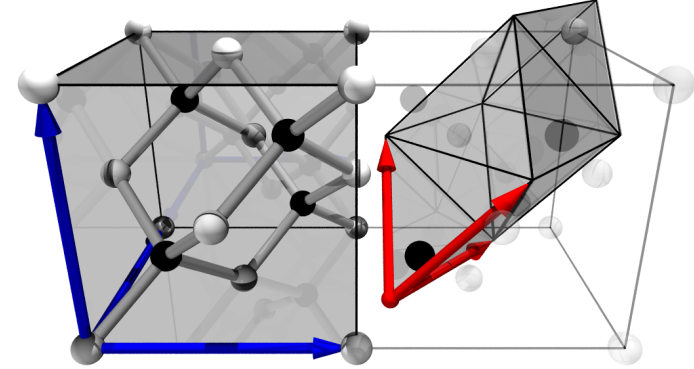

FIG. 1. Bulk structure of diamond/zincblende materials. Light- and dark-shaded atoms correspond, respectively, to those labelled $\alpha$ and $\beta$ in the text. In materials taking the diamond structure, the distinction is purely notational. In those taking the zincblende structure, the distinction corresponds to the presence of two distinct species, with light $(\alpha)$ indicating the cation and dark $(\beta)$ indicating the anion in our convention. On the left of the image, a conventional unit cell is indicated, together with three non-primitive lattice vectors that span it; on the right, three primitive lattice vectors are shown (relative to a shifted origin) together with the primitive unit cell that they span (interatomic bonds omitted for clarity).

another, but it will nevertheless be convenient to retain a nominal distinction. In the zincblende structure, we shall adopt the convention that the atom labelled $\alpha$ corresponds to the cationic species, while the atom labelled $\beta$ corresponds to the anionic species. Note that alternative expressions of the primitive lattice vectors and atomic positions would be equally valid (Ashcroft and Mermin, for example, place one atom at the origin of the primitive cell [2]) but all of the results below have been derived within the convention presented here.

The improper symmetry elements of the diamond and zincblende structures, which will be of importance in ascertaining the chirality of surfaces, comprise a set of mirror planes and glide planes [3]. Both structures exhibit mirror symmetry across planes of $\{110\}$ type - a feature that they share with the $f c c$ crystal structure. Whereas the $f c c$ structure also exhibits mirror symmetry across planes of $\{100\}$ type, however, these are merely glide planes in the diamond structure and are neither mirror nor glide planes in the zincblende structure.

Turning to structural matters, the previous analysis of $f c c$ materials [1] emphasised the existence of close-packed chains of atoms running along the $\langle 110\rangle$ crystallographic directions. These were taken to be the primary structural features whose presence or otherwise at any given surface was to be noted; zig-zag chains of atoms, running along the $\langle 100\rangle$ crystallographic directions, were considered as secondary structural features. In the diamond and zincblende structures, there are no directions in which close-packed chains of atoms exist, so there is no direct analogy to the primary structure of the $f c c$ case; there are, however, zig-zag chains, corresponding to the secondary structure, which in this case align along the $\langle 110\rangle$ crystallographic directions. That is, the same directions that correspond to close-packed primary structure in the $f c c$ crystal structure amount to zig-zag secondary structure for the diamond and zincblende crystal structures. Finally, we note that our previous analysis of the $h c p$ structure introduced the concept of an "interrupted" primary structural feature, where close-packed pairs of atoms were aligned along the $\langle 20 \overline{2} 3\rangle$ crystallographic directions; similarly interrupted close-packed features are present within the diamond structure, lying along the $\langle 111\rangle$ crystallographic directions; in the zincblende structure, these directions link close-packed atoms of differing species.

\section{SURFACE SYMMETRY}

In order to represent the symmetry of all possible surfaces, we proceed by identifying vectors normal to the mirror and glide planes of the bulk structures, and then marking the set of all surface normals perpendicular to these onto a stereographic projection. Since both diamond and zincblende bulk structures feature mirror planes of $\{110\}$ type, we first plot all surfaces whose normal lies perpendicular to any one of the $\langle 110\rangle$ crystallographic directions; these are the so-called "mirror zones", marked as solid red lines on Fig. 2 [4]. Next, for the diamond structure only, we plot (as broken red lines) the so-called "glide zones", corresponding to those surfaces whose normal lies perpendicular to any one of the $\langle 100\rangle$ crystallographic directions, since these are the normals to the glide planes of the bulk. In this manner, the mirror and glide zones of the stereogram indicate those surfaces at which mirror and/or glide planes cut through the surface perpendicularly. In the case of mirror zones, this implies that the unreconstructed surface must necessarily exhibit reflection symmetry across that plane (and, indeed, that any spontaneous symmetry-lowering reconstruction that might occur must lead to domains whose proportions respect the original symmetry). On the glide planes, the situation is less clear, since glide symmetry will only be present at the surface if a suitable glide vector happens to lie within the surface plane. Finally, we plot onto the stereogram any mirror zones possessed by the underlying $f c c$ Bravais lattice that are not mirror or glide zones of the actual crystal structures discussed 

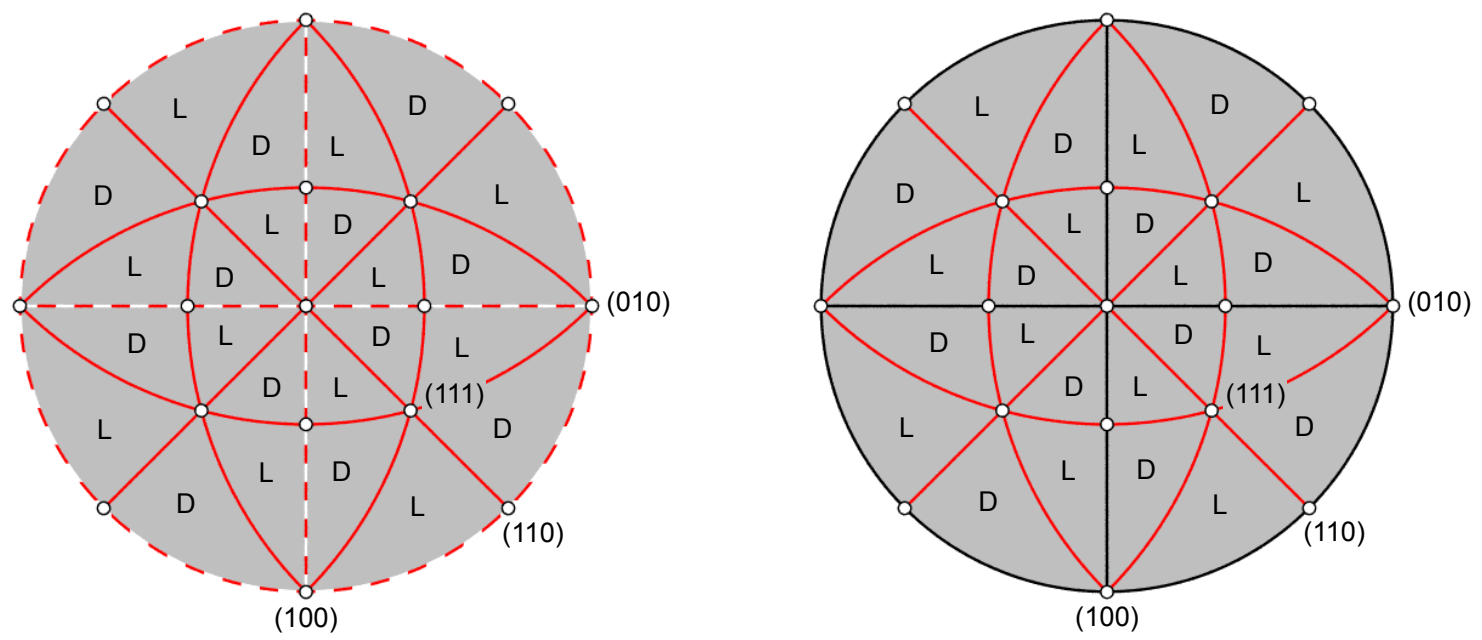

FIG. 2. Stereograms showing symmetry elements at the surfaces of diamond-structure (left) and zincblende-structure (right) materials. Red lines indicate mirror zones, red/white dashed lines indicate glide zones, and small circles highlight surfaces of $\{111\},\{110\}$ and $\{100\}$ type. Labels "D" and "L" relate to chirality arising from truncation of the underlying face-centred cubic lattice in each case.

here; thus, the stereogram for the zincblende case gains zones perpendicular to the $\langle 100\rangle$ crystallographic directions, marked in black, which we shall refer to as "latent zones".

It is clear, then, that the entire stereogram (including its putative reverse side) is divided into a set of 48 "stereographic triangles", bounded in the diamond case by mirror and glide zones, and in the zincblende case by mirror and latent zones. This, in turn, allows us to make robust statements about the symmetry of various surfaces. We can assert, for example, that any surface whose normal lies on none of the zones must necessarily be in some sense chiral, if only because its underlying lattice necessarily lack mirror and glide symmetry. We label the interiors of each triangle "D" or "L", according to a convention introduced previously for the $f c c$ structure [1], in recognition of the chirality that arises solely due to the truncation of the underlying bulk Bravais lattice by the surface plane. Note that this type of chirality swaps sense whenever one crosses any of the three types of symmetry zone; mirror, glide and latent zones all correspond to mirror symmetry in the underlying Bravais lattice. Note also that this type of chirality is absent for those surfaces with normals lying precisely on any of the zones themselves; in such cases, the surface-truncated Bravais lattice possesses mirror symmetry. We denote this latter situation with the symbol "X" (not shown in the figure) to indicate that neither "D" nor "L" would be an appropriate label.

In addition to the chirality associated with truncation of the underlying Bravais lattice, however, it is clear that a second source of chirality must exist. There are certainly surfaces, for instance, in which the surface- truncated Bravais lattice possesses a mirror symmetry but the relative orientation of the $\alpha$ and $\beta$ atoms breaks that symmetry. This added complexity is not present for materials taking the $f c c$ structure, since they possess only a single atom per primitive unit cell, but a similar situation was faced previously in dealing with the $h c p$ structure [1]. Following the same approach used in that case, we augment the labelling of each stereographic triangle with a second chirality symbol (again either "D" or "L") corresponding to this second source of chirality. The second chirality symbol swaps sense when crossing a mirror zone, but not when crossing either a glide zone or a latent zone. The full pattern of chirality, including both sources, is then expressed in Figs. 3 and 4 . Note that a separate diagram is now required for surfaces terminating with either an $\alpha$ or a $\beta$ atom, and recall that for the diamond structure this distinction is purely formal, whilst for the zincblende structure it relates to the physical distinction between cations and anions. Note also that those surfaces whose normals lie precisely on a mirror plane take the designation " $\mathrm{X}$ " for the second chirality symbol, whereas those whose normals lie on glide or latent zones will retain the second chirality symbol appropriate to the adjacent stereographic triangles. Surfaces with normals lying on a mirror zone thus acquire the designation XX and may be described as "reflexive", indicating that they are entirely achiral due to possession of a mirror plane.

Returning to the distinction between atoms designated $\alpha$ and $\beta$, the most important consequence that we have not yet strongly emphasised is that each surface may possess up to two possible ideal (i.e. unreconstructed) terminations, depending upon the height at which the surface 

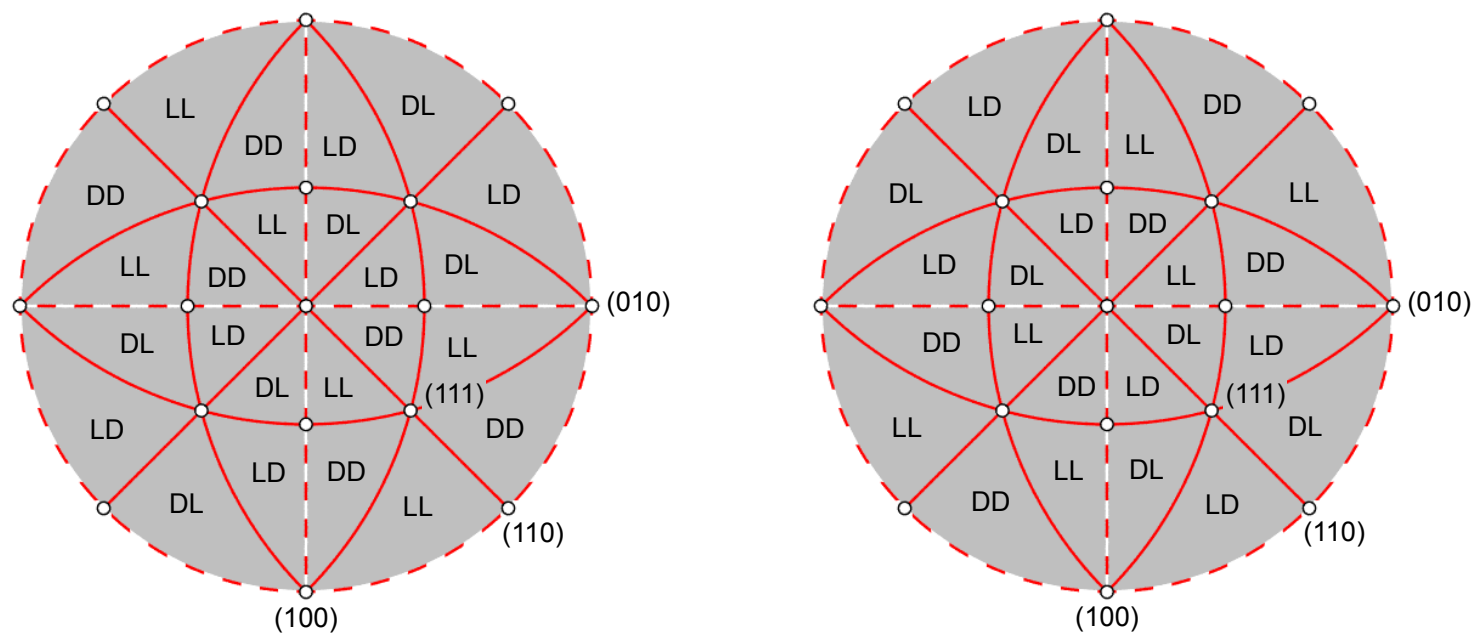

FIG. 3. Stereograms showing symmetry elements of diamond-structure surfaces. The diagram on the left relates to surfaces terminating with atoms labelled $\alpha$, and that on the right relates to surfaces terminating with atoms labelled $\beta$ (see text). Red lines indicate mirror zones, red/white dashed lines indicate glide zones, and small circles highlight surfaces of $\{111\},\{110\}$ and $\{100\}$ type. Labels "DD", "LL", "DL" and "LD" indicate two forms of chirality, the first arising due to the truncation of the underlying face-centred cubic lattice, and the second from the disposition of the atomic basis relative to the truncated lattice.
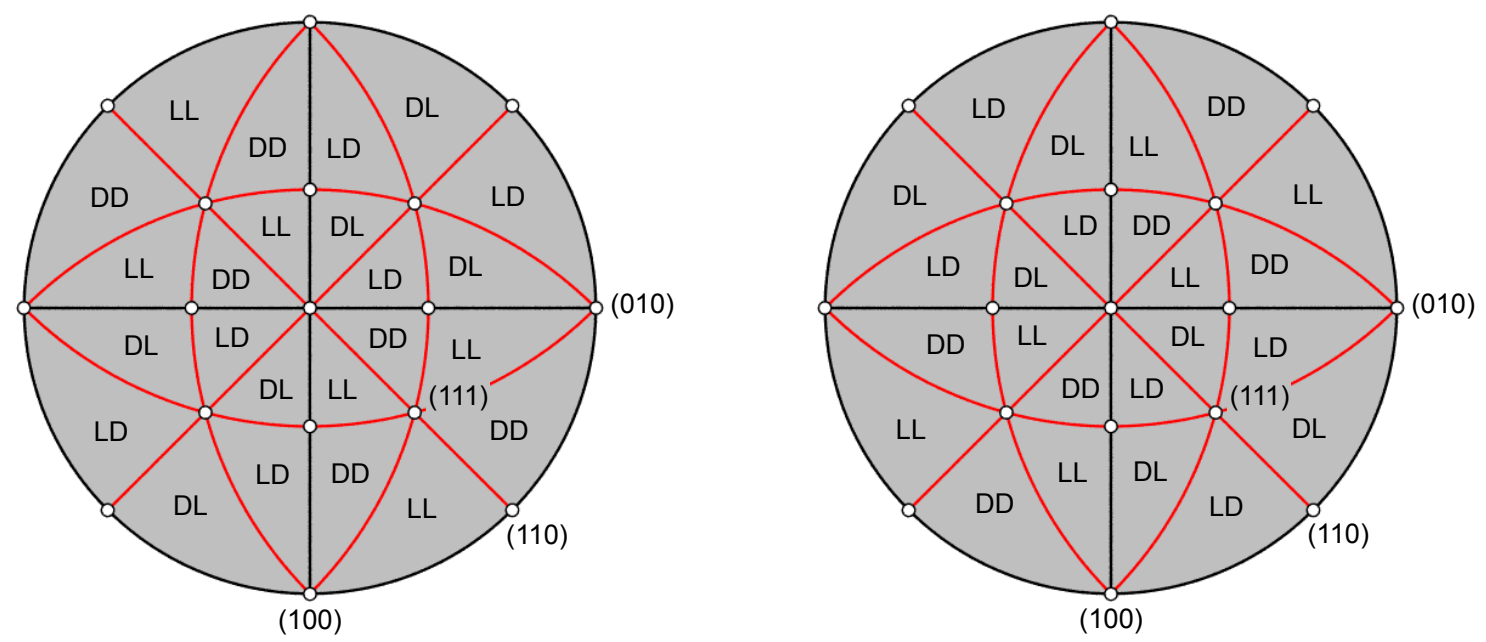

FIG. 4. Stereograms showing symmetry elements of zincblende-structure surfaces. The diagram on the left relates to surfaces terminating with atoms labelled $\alpha$ (cations) and that on the right relates to surfaces terminating with atoms labelled $\beta$ (anions). Red lines indicate mirror zones, black lines indicate latent zones, and small circles highlight surfaces of $\{111\},\{110\}$ and $\{100\}$ type. Labels "DD", "LL", "DL" and "LD" indicate two forms of chirality, the first arising due to the truncation of the underlying face-centred cubic lattice, and the second from the disposition of the atomic basis relative to the truncated lattice.

plane cuts through the bulk structure. In the general case, the termination featuring $\alpha$ atoms in the outermost layer will have an entirely different structure from that featuring $\beta$ atoms in the outermost layer. Special cases arise, however, when the outermost layer contains atoms of both $\alpha$ and $\beta$ type and hence there will be only a single termination - a situation we shall describe as "unitermination". To enumerate the uniterminated surfaces, we must first identify the set of all possible vectors linking $\alpha$ atoms to $\beta$ atoms in the bulk structure. For both the di- amond and zincblende cases, this set may be represented simply as $\frac{1}{4}[p q r]$, with $p, q$ and $r$ being odd integers. The requirement that a particular surface plane, having Miller indices $(h k l)$, contains such a vector (and hence corresponds to a uniterminated surface) amounts simply to

$$
h p+k q+l r=0
$$

with $h, k$ and $l$ subject to the usual constraint that they 
share no common factor. In effect, one could visualise a dense mesh of "unitermination zones", corresponding to the normals of the uniterminated surfaces, each one perpendicular to a different $[p q r]$ crystallographic direction.

For the surfaces of materials taking the diamond structure, the significance of unitermination is essentially academic, but for the zincblende materials, it provides the formal definition of a non-polar surface (as discussed further below). Note that the condition of unitermination, for either bulk structure, implies the conflation either of a DD termination with a DL one, or of an LL termination with an LD one, which in turn implies that the second chirality symbol must reduce to $\mathrm{X}$ for all uniterminated surfaces. Where unitermination zones intersect a latent zone, therefore, we expect surfaces whose two-component chirality symbol is simply XX (because the first chirality symbol reduces to $\mathrm{X}$ here also) which leads one to believe that such surfaces must be entirely achiral. In the diamond case, this is indeed true. At this point, however, we must recall that the distinction between $\alpha$ and $\beta$ atoms is a real one in the zincblende structure, so that although the arrangement of generic atoms in the ideal surface would indeed be achiral in all these cases, this no longer holds when the actual identity of those atoms is taken into account. We therefore note the uniterminated latent surfaces as special cases.

For surfaces that are not uniterminated, it is necessary to keep track of the different possible terminations in all cases. Once again following the approach developed for the surfaces of $h c p$ materials [1], we do so by adopting the convention that terminations whose two-component chirality label is either DD or LL will be designated $\tau$ and those whose two-component chirality label is either DL or LD will be designated $\bar{\tau}$. For surfaces sharing symmetryrelated Miller indices, terminations designated $\tau$ will be either identical to one another or mirror images of each other; the same is true of terminations designated $\bar{\tau}$; in contrast, a termination designated $\tau$ will be neither identical to, nor a mirror image of, a termination designated $\bar{\tau}$. Special cases arise for uniterminated surfaces, where $\tau / \bar{\tau}$ will be replaced with the designation $u$, and for those surfaces whose normals lie on mirror or glide zones.

Along the glide zones of the diamond-structure stereogram lie the normals of surfaces having some quite unusual properties. Wherever glide zones are intersected by unitermination zones, one finds the normal of a surface possessing glide symmetry; we shall describe such a surface as "glissadic". The $\{110\}$ surfaces are familiar examples, but the complete family of glissadic surfaces comprises all those of $\{p q 0\}$ type, where $p$ and $q$ are both odd. All other surfaces along the glide zone possess two distinct terminations that are precise mirror images of one another; this family we shall describe as "racemic", and they comprise all surfaces of $\{p m 0\}$ type, with $p$ odd and $m$ even. In the case of zincblende structure surfaces, the distinction between $\alpha$ and $\beta$ atoms means that oth-

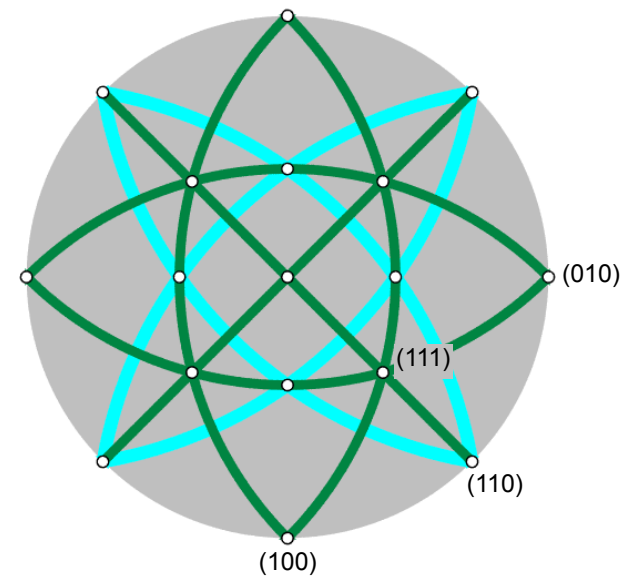

FIG. 5. Stereogram showing structural features elements found at the surfaces of diamond- or zincblende-structure materials. Interrupted primary zones are depicted in cyan, with secondary zones marked in green.

erwise glissadic surfaces are found to be chiral (as noted above) while otherwise racemic surfaces are doubly chiral.

Finally, we note the surfaces of highest overall symmetry, which arise at the intersections of mirror and glide zones. For the diamond structure, these comprise the $\{111\}$ surfaces, each possessed of three mirror planes and a three-fold rotational axis; the $\{001\}$ surfaces, each with two mirror planes and a four-fold rototruncation axis [5]; and the $\{110\}$ surfaces, each with one mirror plane, one glide symmetry, a two-fold rotational axis and pure truncation symmetry. For the zincblende structure, the $\{001\}$ surfaces lack rototruncation symmetry, while the $\{110\}$ surfaces lack both glide and rotational symmetries; the $\{111\}$ surfaces exhibit the same symmetries as for the diamond structure.

\section{SURFACE STRUCTURE}

In order to represent the structure of all possible surfaces, we plot a stereographic projection of zones perpendicular to the crystallographic directions associated with key structural features of the bulk materials. As discussed above, the primary structural feature associated with close-packed chains of atoms is absent from both the diamond and zincblende bulk structures, but there are interrupted primary features (i.e. close-packed pairs of atoms) in the $\langle 111\rangle$ crystallographic directions, and secondary features (i.e. zig-zag chains of atoms) in the $\langle 110\rangle$ crystallographic directions. These are depicted stereographically in Fig. 5.

Immediately, we can identify a variety of surface structural categories. Where the surface normal lies on pre- 
cisely one of the interrupted primary zones, the structure may be described as "geminal", indicating the existence of a pair of close-packed atoms lying within the surface plane; relaxation will almost certainly lead to a buckling, but we retain our formal categorisation on the basis of the ideal structure. The general condition for such a surface may be shown to dictate Miller indices $\{\mathrm{hkl}\}$ satisfying $|h|-|k|-|l|=0$, or an equivalent permutation.

Where the surface normal lies at the intersection of two interrupted primary zones, on the other hand, the structure may be classified as of "meandering row" type, indicating that close-packed pairs of atoms zig-zag across the surface (with all atoms in the zig-zag at the same height, neglecting relaxation). This situation arises only for surfaces of $\{110\}$ type, but it is worth noting that there exists a wider class of surfaces - those lying elsewhere on one of the secondary zones - for which something like a meandering row exists, but rotated by some angle around its axis; these "canted" meandering rows are found for all surfaces of $\{s s t\}$ type, with no restriction on the parity of $s$ or $t$ (other than that they cannot both be even). Notable amongst these surfaces are those of $\{112\}$ type, whose normals each lie at the intersection of an interrupted primary zone and a secondary zone; here the surface is not only geminal (i.e. exhibits pairs of atoms in the same layer) but also features a canted meandering row (with a cant angle of $54.74^{\circ}$ ).

Other notable surfaces, from the structural perspective, occur at the intersections of secondary zones with each other. Three such zones intersect at the normals of $\{111\}$ surfaces, giving rise to a structure in which three canted meandering rows run across the surface, separated by $60^{\circ}$ and each canted by an angle of $35.26^{\circ}$ about its own axis. Two secondary zones intersect at the normals of $\{100\}$ surfaces, giving rise to a structure in which two canted meandering rows run across the surface at rightangles to one another, each canted over at right-angles to the surface normal.

The lack of non-interrupted primary zones, in the presently considered cases, means that no counterparts exist for the flat and stepped categories of surface discussed for the metallic structure[1]. Kinked surfaces, however, in which atoms within the top layer have no in-plane nearest neighbours, are found wherever a surface normal lies off all the zones marked in Fig. 5.

\section{SYNTHESIS OF SURFACE SYMMETRY AND STRUCTURE FOR DIAMOND}

Bringing together the analysis of symmetry and structure from the preceding two sections, the stereograms presented in Figs. 6 and 7 allow us to identify a limited range of permissible combinations. Kinked surfaces can exist across all the symmetry classes identified above, with the sole exception of the reflexive-glissadic type; the

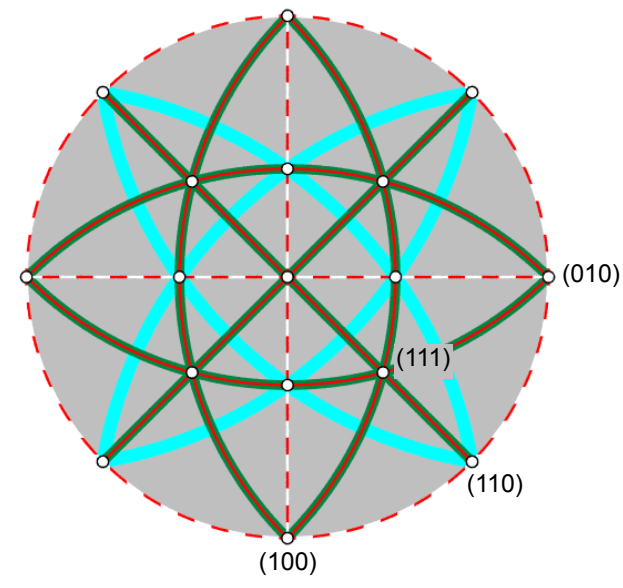

FIG. 6. Stereogram combining structure and symmetry information for surfaces of diamond-structure materials. The colour scheme for zones is as described in preceding figures.

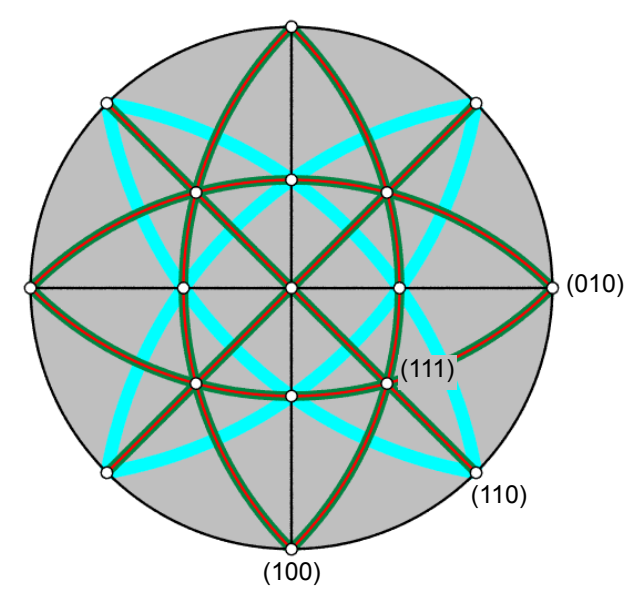

FIG. 7. Stereogram combining structure and symmetry information for surfaces of zincblende-structure materials. The colour scheme for zones is as described in preceding figures.

latter symmetry class can only occur in combination with the meandering row structural feature. Geminal surfaces can fall into either the singly chiral or the uniterminatedreflexive symmetry classes, but no others. In the case of materials taking the diamond structure, eleven welldefined combinations results, as shown in Fig. 8. We shall retain these categories for the zincblende structure, even though some of the symmetries are obscured by the presence of two atomic species. Accordingly, we make the following observations:

Bayonet Kinked Surfaces

These are the six $\{001\}$ surfaces, which in the diamond structure each possess two mirror planes (perpendicular to $\langle 110\rangle$ axes) together with a four-fold rototruncation symmetry [6]. The four degenerate terminations associated with each surface orientation are thus related by 


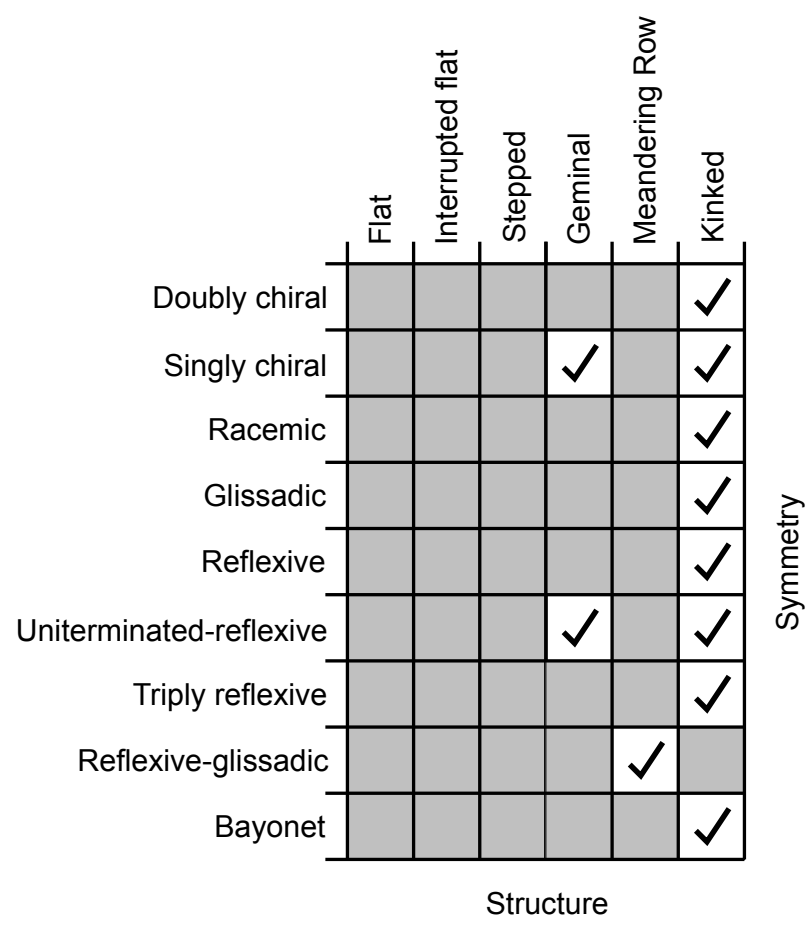

FIG. 8. Permitted combination of structure and symmetry.

rotation (and all are designated $\mathrm{XX}_{o}$ in the notation of reference [1]). In the zincblende structure, these surfaces lack the rototruncation symmetry element.

Reflexive-Glissadic Meandering Row Surfaces

These comprise the twelve $\{110\}$ surfaces, each displaying, in the diamond case, a single mirror plane (perpendicular to a $\langle 110\rangle$ axis) and a single glide plane (perpendicular to a $\langle 100\rangle$ axis). All are uniterminated (designated $\mathrm{XX}_{u}$ ) and feature a meandering row whose axis lies within the glide plane. In the zincblende case, the glide symmetry is lacking, and the atoms within the meandering row alternate between cations and anions.

Triply-Reflexive Kinked Surfaces

These are the eight $\{111\}$ surfaces, each possessing three mirror planes (perpendicular to $\langle 110\rangle$ axes) and no glide symmetry, whether for diamond or zincblende structure materials. Since they display neither pure nor compound truncation symmetry, the two distinct terminations possible for a single surface orientation are unrelated by symmetry and non-degenerate (designated $\mathrm{XX}_{\tau}$ and $\mathrm{XX}_{\bar{\tau}}$ ).

Uniterminated-Reflexive Geminal Surfaces

These comprise the twenty-four $\{112\}$ surfaces, each possessing a single mirror plane (perpendicular to a $\langle 110\rangle$ axis) and displaying no glide symmetry, for both diamond and zincblende structure materials. The presence of pure truncation symmetry implies that only a single unique termination exists for each specific surface orientation (designated $\mathrm{XX}_{u}$ ) which in these instances features pairs of close-packed atoms within the ideal surface plane; in the zincblende case, one will be of the cationic species, the other anionic.

Uniterminated-Reflexive Kinked Surfaces

This category consists of an infinite number of surfaces with indices conforming to the pattern $\{2 p+12 p+12 q\}$ with $p$ and $q$ integers (excepting the $\{110\}$ and $\{112\}$ special cases described above). For both the diamond and zincblende cases, all possess a single mirror plane (perpendicular to a $\langle 110\rangle$ axis) and display no glide symmetry, while the presence of pure truncation symmetry implies that only a single unique termination exists for each specific surface orientation (designated $\mathrm{XX}_{u}$ ).

\section{Reflexive Kinked Surfaces}

This category comprises the infinite set of surfaces with indices conforming to the pattern $\{p p 2 q+1\}$ with $p$ and $q$ integers (excepting the $\{001\}$ and $\{111\}$ surfaces described above). Each such surface, whether of the diamond or zincblende structure, possesses a single mirror plane (perpendicular to a $\langle 110\rangle$ axis) and displays no glide symmetry. The absence of any form of truncation symmetry implies that each surface orientation supports two non-degenerate terminations, unrelated by symmetry (designated $\mathrm{XX}_{\tau}$ and $\mathrm{XX}_{\bar{\tau}}$ ).

\section{Glissadic Kinked Surfaces}

This category consists of an infinite number of surfaces with indices conforming to the pattern $\{2 p+12 q+10\}$ with $p$ and $q$ unequal integers. In the diamond structure, all possess a single glide plane (perpendicular to a $\langle 100\rangle$ axis) and the presence of truncation symmetry implies that only a single unique termination exists for each surface orientation (designated $\mathrm{XX}_{\varnothing}$ ). In the zincblende structure, the glide symmetry is absent and these surfaces remain chiral.

\section{Racemic Kinked Surfaces}

This category comprises the infinite set of surfaces with indices conforming to the pattern $\{2 p+12 q 0\}$ with $p$ and $q$ integers. Each such surface, within the diamond structure, is defined by reflexotruncation symmetry, lacking both mirror and glide planes, and hence a single orientation supports two degenerate terminations related by reflection (designated $\mathrm{XD}_{o}$ and $\mathrm{XL}_{o}$ ). For surfaces of the zincblende structure, this reflexotruncation symmetry is absent and the two terminations are non-degenerate; each may be found with either cationic or anionic termination, albeit at different points in the stereogram.

\section{Singly-Chiral Geminal Surfaces}

This category consists of an infinite number of surfaces with indices conforming to the pattern $\{p q p+q)$ with $p$ and $q$ different positive integers. For both diamondand zincblende-structure materials, all lack both mirror and glide symmetry, but the presence of truncation symmetry implies that only a single unique termination (featuring pairs of close-packed atoms within the ideal surface plane) exists for each surface orientation (designated $\mathrm{DX}_{u}$ or $\mathrm{LX}_{u}$ ). 
Singly-Chiral Kinked Surfaces

This category comprises the infinite set of surfaces with indices conforming to the pattern $\{2 p+12 q+12 r\}$ with $p, q$ and $r$ integers. Each such surface lacks both mirror and glide symmetry, for both the diamond and zincblende cases, while the presence of truncation symmetry ensures that it supports only a single unique termination for a given surface orientation (designated $\mathrm{DX}_{u}$ or $\mathrm{LX}_{u}$ ).

Doubly-Chiral Kinked Surfaces

This category encompasses the infinity of surfaces whose indices fail to conform to any of the patterns discussed above. All such surfaces lack mirror, glide and any form of truncation symmetry, whether within the diamond or the zincblende structure. Any specific surface orientation therefore supports two non-degenerate terminations, unrelated by symmetry (designated either $\mathrm{DD}_{\tau}$ and $\mathrm{DL}_{\bar{\tau}}$, or $\mathrm{LL}_{\tau}$ and $\mathrm{LD}_{\bar{\tau}}$ ). In the zincblende case, cationic or anionic terminations may be found for each symmetry symbol, distributed across the stereogram.

\section{ON THE POLARITY OF ZINCBLENDE SURFACES}

The only fundamental difference between the diamond and zincblende structures is that one features but a single element, while the other alternates cationic and anionic species. This simple point of distinction, however, implies significant consequences for the stability of zincblende surfaces. Specifically, while there exist some ideal zincblende surface orientations in which cationic and anionic atoms occur in equal proportion, there are many more in which their numbers fail to balance; the former category are said to be non-polar and the latter polar. The key consequences of surface polarity were articulated most clearly by Harrison [7, 8] and are summarised in the following paragraph.

All ideal semiconductor surfaces necessarily feature partially occupied dangling bonds, and surface stability revolves around eliminating these (where possible) through relaxation and/or reconstruction. For instance, relaxation may be associated with the redistribution of electrons between dangling bonds, such that some dangling bonds become completely filled while others become entirely empty. In other cases, reconstruction (e.g. dimerisation or trimerisation) may allow neighbouring dangling bonds to form true interatomic bonds, complete with filled/empty bonding/antibonding orbitals. In the case of a non-polar surface, the number of electrons present within the original partially occupied dangling bonds is precisely sufficient to allow relaxation and/or reconstruction to perfectly eliminate partial occupancy without either accumulating a net surface charge or altering the surface stoichiometry. In the case of a polar surface, however, the opposite will be true. A polar surface must either reconstruct in non-stoichiometric man- ner, or acquire a net electronic charge - the former will be limited by availability of one atomic species in excess of the other, while the latter entails an electromagnetic instability that tends to infinity as the surface area increases. This is not to say that polar surfaces are impossible. They can certainly exist, for example, as facets of limited spatial extent, where the net positive charge (or excess of cations) needed on one facet can readily be balanced by a net negative charge (or excess of anions) on a differently oriented nearby facet. Nevertheless, identifying which surfaces are polar and which are not is of no little importance in the study of semiconductor surfaces.

As noted above, the condition for polarity of a zincblende material turns out to be synonymous with the condition of unitermination. That, in turn, implies that the only non-polar zincblende surfaces are those of $\{110\}$ type, those of $\{112\}$ type, and those whose indices conform to the pattern $\{p q p+q)$ with $p$ and $q$ different positive integers, for instance the chiral $\{123\},\{134\}$ and $\{235\}$ surfaces. Of these categories, the first type display both mirror and glide symmetry, the second only mirror symmetry, and the third are intrinsically chiral. The $\{110\}$ surfaces of zincblende semiconductor surfaces are, indeed, typically highly stable, and have been very well studied over many decades. The $\{112\}$ surfaces, on the other hand, have been investigated much less often, but do hold considerable potential (see, for example, Geelhaar et al[9]).

\section{CONCLUSIONS}

The structure and symmetry of diamond- and zincblende-structure materials have been examined within a stereographic framework used previously only for metallic systems. Circumstances leading to surface chirality have been outlined, and key structural features identified. The property of unitermination has been discussed, including its link to the polarity or otherwise of zincblende surfaces. In all, eleven distinct structuresymmetry categories have been described, which it may be hoped will prompt future experimental and computational studies.

\section{ACKNOWLEDGMENTS}

The author is indebted to Dr Stephanie Pratt, with whom the stereographic approach to surface symmetry and structure was originally devised.

[1] Jenkins, S.J.; Pratt, S.J. Beyond the Surface Atlas: a Roadmap and Gazetteer for Surface Symmetry and Structure. Surf. Sci. Rep. 2007, 62, 373-429. 
[2] Ashcroft, N.W.; Mermin, N.D. Solid State Physics (Saunders, 1976)

[3] The definition of a mirror plane is simple - reflection of the crystal structure across such a plane must result in a structure identical with the original. The concept of a glide plane is more involved - reflection across such a plane leads to a structure that is identical with the original only after translation through a specific vector parallel to the glide plane. Mirror symmetry exclusively maps $\alpha$ atoms onto $\alpha$ atoms and $\beta$ atoms onto $\beta$ atoms; glide symmetry interchanges $\alpha$ atoms with $\beta$ atoms throughout.

[4] The term "zone" is used in crystallography to indicate a set of planes whose normals all lie perpendicular to a common direction known as the "zone axis". Strictly speaking, a line drawn on a stereogram to indicate such a set of planes (in our case, surface planes) is properly known as the "trace" of that zone. Since there is a one-to-one correspondence between a zone and its trace, however, it is rarely necessary to draw such a fine distinction, and we have therefore chosen to refer throughout to traces as zones. It is, perhaps, unfortunate that the word zone is suggestive of a two-dimensional area, rather than a onedimensional line, but short of redefining crystallographic nomenclature this is practically unavoidable.

[5] The rototruncation symmetry element is defined when a screw axis of the bulk lies perpendicular to the surface.
The corresponding screw operation is evidently not a symmetry of the surface, since it changes the height of the surface, but the addition or subtraction of an appropriate number of atomic layers (what we might term the pure truncation operation) combines with the screw to constitute a valid symmetry operation. Combination of the truncation operation with a vertically oriented bulk glide axis, on the other hand, results in the so-called reflexotruncation symmetry element, present in the racemic surfaces described above.

[6] The similarity of the rototruncation operation to the action implied by a so-called bayonet fitting (e.g. for a light bulb) prompted us previously [1] to coin the appellation "bayonet" to describe the symmetry in such cases.

[7] Harrison, W.A. Surface Reconstruction on Semiconductors. Surf. Sci. 1976, 55, 1-19.

[8] Harrison, W.A. Theory of Polar Semiconductor Surfaces. J. Vac. Sci. Technol. 1979, 16, 1492-1496.

[9] Geelhaar, L.; Marquez, J.; Jacobi, K.; Kley, A.; Ruggerone, P.; Scheffler, M. A Scanning Tunneling Microscopy Study of the GaAs(112) Surfaces. Microelectron. J. 1998, 30, 393-396. 


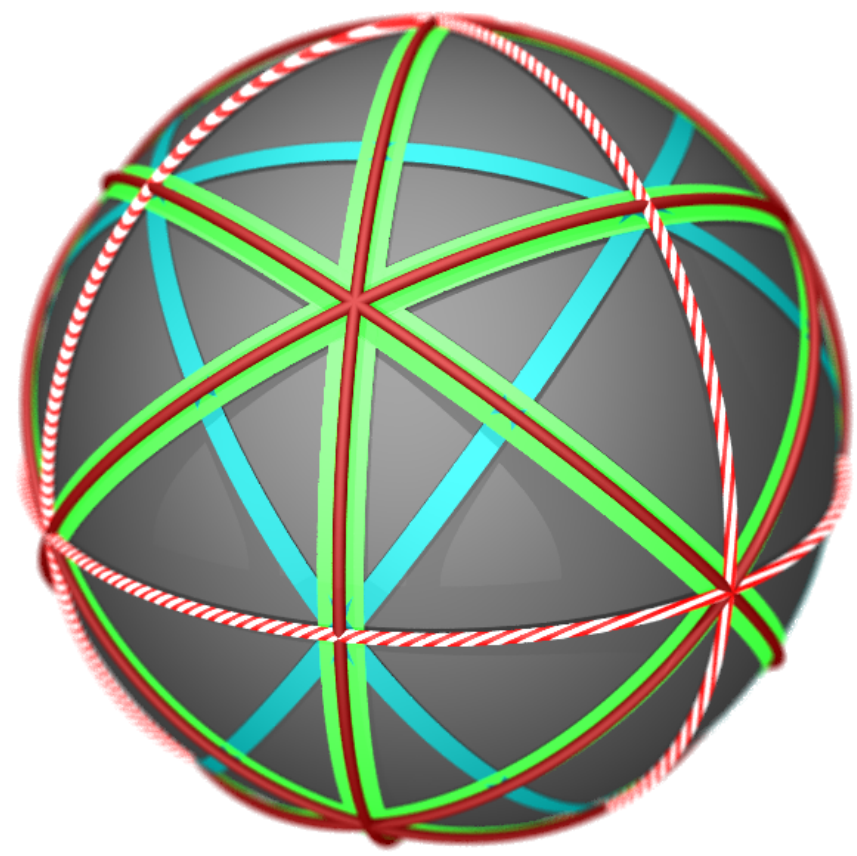

FIG. 9. Table of Contents Graphic - The symmetry and structure of surfaces may be represented by means of great circles inscribed upon a sphere (or curved lines on a stereographic projection of the sphere). Here, the red and red-striped lines show mirror and glide symmetries, while the cyan and green ones depict specific structural features. 